\title{
Risk of fragility fracture among patients with sarcoidosis: a population-based study 1976-2013—supplementary presentation
}

\author{
P. Ungprasert ${ }^{1}$ (D) C. S. Crowson ${ }^{1} \cdot$ E. L Matteson ${ }^{1}$
}

Received: 17 March 2017 / Accepted: 2 May 2017 / Published online: 22 March 2018

(C) International Osteoporosis Foundation and National Osteoporosis Foundation 2018

\begin{abstract}
Incidence of fragility fracture of a population-based cohort of 345 patients with sarcoidosis was compared with age- and sex-matched comparators. The incidence of fragility fracture was higher among patients with sarcoidosis with a hazard ratio (HR) of 2.18 .
\end{abstract}

\section{Introduction}

Several chronic inflammatory disorders increase the risk of fragility fracture. However, little is known about the risk of fragility fracture in patients with sarcoidosis.

\section{Methods}

This study was conducted using a previously identified population-based cohort of 345 patients with incident sarcoidosis from Olmsted County, Minnesota. Diagnosis of sarcoidosis required physician diagnosis supported by biopsy showing non-caseating granuloma, radiographic evidence of intrathoracic sarcoidosis, and compatible clinical presentations without evidence of other granulomatous diseases. Sex- and age-matched subjects randomly selected from the same underlying population were used as comparators. Medical records of cases and comparators were reviewed for baseline characteristics and incident fragility fracture.

Electronic supplementary material The online version of this article (https://doi.org/10.1007/s00198-017-4074-4) contains supplementary material, which is available to authorized users.

P. Ungprasert

p.ungprasert@gmail.com

1 Mayo Clinic, Rochester, MN, USA

\section{Results}

Fragility fractures were observed in 34 patients with sarcoidosis, corresponding to a cumulative incidence of $5.6 \%$ at 10 years, while 18 fragility fractures were observed among comparators for a cumulative incidence of $2.4 \%$ at 10 years. The HR of fragility fractures among cases compared with comparators was 2.18 (95\% confidence interval [CI], 1.233.88). The risk of fragility fracture by site was significantly higher among patients with sarcoidosis and was due to a higher rate of distal forearm fracture (HR 3.58; 95\% CI $1.53-8.40)$. Statistically non-significant increased risk was also observed in proximal femur (HR 1.66; 95\% CI 0.45-6.06) and proximal humerus (HR 3.27; 95\% CI 0.66-16.21). Risk of vertebral fracture was not increased (HR 1.00; 95\% CI 0.32-3.11).

\section{Conclusion}

Patients with sarcoidosis have an increased risk of fragility fracture which is primarily driven by the higher incidence of distal forearm fracture [1].

\section{Reference}

1. Ungprasert P, Crowson CS, Matteson EL (2017) Risk of fragility fracture among patients with sarcoidosis: a population-based study 1976-2013. Osteoporos Int 28:1875-1879. https://doi.org/10.1007/ s00198-017-3962-y 